\title{
Zener tunneling in two-dimensional photonic lattices
}

\author{
Valery S. Shchesnovich, ${ }^{1}$ Solange B. Cavalcanti, ${ }^{1}$ Jandir M. Hickmann, ${ }^{1}$ and Yuri S. Kivshar ${ }^{2}$ \\ ${ }^{1}$ Instituto de Física, Universidade Federal de Alagoas, Maceió AL 57072-970, Brazil \\ ${ }^{2}$ Nonlinear Physics Center and Centre for Ultra-high Bandwidth Devices for Optical Systems (CUDOS), Research School of Physical \\ Sciences and Engineering, Australian National University, Canberra Australian Capital Territory 0200, Australia
}

(Received 5 June 2006; revised manuscript received 1 August 2006; published 2 November 2006)

\begin{abstract}
We discuss the interband light tunneling in a two-dimensional periodic photonic structure, as studied recently in experiments for optically induced photonic lattices [Trompeter et al., Phys. Rev. Lett. 96, 053903 (2006)]. We identify the Zener tunneling regime at the crossing of two Bloch bands, which occurs in the generic case of a Bragg reflection when the Bloch index crosses the edge of the irreducible Brillouin zone. Similarly, higher-order Zener tunneling involves four Bloch bands when the Bloch index passes through a high-symmetry point on the edge of the Brillouin zone. We derive simple analytical models that describe the tunneling effect, and calculate the corresponding tunneling probabilities.
\end{abstract}

DOI: 10.1103/PhysRevE.74.056602

PACS number(s): 42.25.Bs, 42.82.Et, 42.65.Wi

\section{INTRODUCTION}

Zener tunneling in tilted periodic potentials is an intriguing physical phenomenon which occurs when the energy difference imposed on the period by a linear potential becomes of the order of the energy gap between two nearest Bloch bands [1]. A simple model studied independently by Zener [1], Landau [2], and Majorana [3] captures the essence of this phenomenon. The well-known physical examples include electrical breakdown in Zener diodes [4], electrical conduction in nanotubes [5] and superlattices [6], pair tunneling in Josephson junctions [7], tunneling of the BoseEinstein condensate in optical lattices [8,9], and an optical analog of tunneling in waveguide arrays and photonic crystals $[10,11]$.

Recent observation of Zener tunneling in two-dimensional photonic lattices [11] calls for a nontrivial generalization of the Landau-Zener-Majorana system, since the latter describes only the tunneling at an avoided crossing of two Bloch bands. In two dimensions the situation of more than two energy levels with comparable energy gaps between them is generally unavoidable. This is due to the fact that in higher dimensions there exist more than one distinct (i.e., not equivalent) Bragg reflection planes. Thus, at least in some cases an $n$-level system with $n>2$ must be invoked to describe Zener tunneling in a two-dimensional lattice. In this paper we analytically study all possible scenarios of Zener tunneling in two-dimensional square lattices. To this end we invoke the so-called shallow lattice approximation (see the definition below). Our results can be applied to the tunneling in two-dimensional photonic lattices observed in Ref. [11].

Bloch oscillations and Zener tunneling in twodimensional lattices have been studied numerically in Refs. $[12,13]$. However, a complete analysis of all possible cases was not presented. In addition, nonlinear effects of Zener tunneling of Bose-Einstein condensate in two-dimensional optical lattices has been considered recently [14] where it was shown that the modulational instability of Bloch waves results in an asymmetry of the resonant upper-to-lower band vs lower-to-upper band tunneling (see also Refs. [15-17] for other nonlinear effects of tunneling in one-dimensional lat- tices). In our case, nonlinearity is negligible and we restrict ourselves to the linear Zener tunneling.

The paper is organized as follows. In Sec. II we consider the general model for the beam propagation in a photonic lattice and discuss the shallow-lattice approximation. Section III contains the derivation of the Landau-Zener-Majorana type models for Zener tunneling in a shallow lattice. Conclusions and perspectives are summarized in Sec. IV.

\section{MODEL AND SHALLOW-LATTICE APPROXIMATION}

We describe the propagation of an optical beam by the paraxial equation for the normalized electric field envelope $E$,

$$
i \frac{\partial E}{\partial \zeta}+\frac{1}{2}\left(\frac{\partial^{2} E}{\partial \xi_{1}^{2}}+\frac{\partial^{2} E}{\partial \xi_{2}^{2}}\right)+\varkappa \Delta n(\xi) E=0,
$$

where $\boldsymbol{\xi}=\left(\xi_{1}, \xi_{2}\right)$ are dimensionless transverse coordinates: $\xi_{1}=(2 \pi / d) x, \xi_{2}=(2 \pi / d) y ; \zeta=2 \pi \lambda /\left(n_{0} d^{2}\right) z$ is the dimensionless propagation distance, with $\lambda$ being the wavelength in vacuum, $n_{0}$ the bulk refractive index, and $d$ the lattice period; $\varkappa=\gamma n_{0}(d / \lambda)^{2}$ with $\gamma$ being the nonlinear coefficient. The total induced refractive index pattern $\Delta n(\xi)$ is given as follows:

$$
\Delta n=\frac{I_{g}(\boldsymbol{\xi})+I_{m}(\boldsymbol{\xi})}{1+I_{g}(\boldsymbol{\xi})+I_{m}(\boldsymbol{\xi})},
$$

where the potential

$$
I_{g}(\boldsymbol{\xi})=A^{2} \cos ^{2}\left(\frac{\xi_{1}}{2}\right) \cos ^{2}\left(\frac{\xi_{2}}{2}\right)
$$

is due to an optical lattice and the additional potential

$$
I_{m}(\boldsymbol{\xi})=\frac{B}{2}\left[1+\tanh \left(\kappa \boldsymbol{e}_{R} \cdot \boldsymbol{\xi}\right)\right],
$$

where $\kappa=d /(2 \pi \eta)$ describes the refractive index ramp in the direction $\boldsymbol{e}_{R}$ (the notations correspond to those of Ref. [11]).

The shallow-lattice approximation corresponds to the condition $x \Delta n \ll 1$ [see Eq. (1)]. For instance, in the experiment 


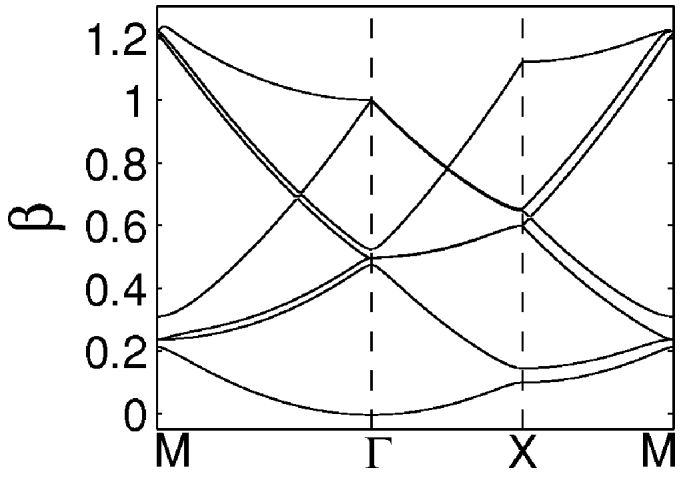

FIG. 1. Bloch band structure (the first seven bands) corresponding to the lattice of Eq. (5). Here $V_{0}=0.05$ and $\varepsilon=1$.

reported in Ref. [11] the induced optical lattice had $x=21.59$. Thus to have a weak-lattice case within the same experimental parameters one should use a weak $\mathrm{cw}$ laser beam satisfying the condition $A^{2} \ll 1 / \varkappa \approx 0.05$.

Assuming that a weak ramp field $(B \ll 1)$ changes slowly over the lattice period, i.e., $2 \pi \kappa B=B d / \eta \ll A^{2}$, and therefore the Bloch band structure is preserved [18-20], we obtain in this case

$$
x \Delta n=x I_{g}(\boldsymbol{\xi})+\frac{1}{2} x B+\boldsymbol{\alpha} \cdot \boldsymbol{\xi},
$$

where $\boldsymbol{\alpha}=\varkappa B \kappa \boldsymbol{e}_{R} / 2=\gamma n_{0} B d^{3} /\left(4 \pi \lambda^{2} \eta\right) \boldsymbol{e}_{R}$. Further discussion of the validity of the Landau-Zener-Majorana models derived below is given in the next section.

Instead of $x I_{g}(\xi)$, we will use the combined potential

$$
V=V_{0}\left[\cos \left(\xi_{1}\right)+\cos \left(\xi_{2}\right)+\varepsilon \cos \left(\xi_{1}\right) \cos \left(\xi_{2}\right)\right]
$$

where $V_{0}=x A^{2} / 4$, and $\varepsilon$ is a parameter that enables us to establish the effect of the nonseparability of the photonic lattice $I_{g}(\boldsymbol{\xi})$ on the tunneling probability. We also drop the insignificant constant terms $\varkappa B / 2$ and $\varkappa A^{2} / 4$. The weak ramp condition now reads $|\boldsymbol{\alpha}| \ll V_{0}$.

The lattice (3), also called the "quantum antidot" in the field of Bose-Einstein condensates [13], corresponds to $\varepsilon=1$. Its band structure for $V_{0}=0.05$ is shown in Fig. 1 .

\section{SIMPLE MODELS FOR ZENER TUNNELING}

An optical beam propagating in the lattice experiences a strong reflection at the resonant Bragg planes (lines in two dimensions), i.e., the Bragg planes which are defined by the reciprocal lattice vectors for which the lattice potential has nonzero Fourier components (see, e.g., Refs. [21,22]). Let us first briefly recall the basic theory. In the Fourier space the equation for the periodic Bloch wave $\varphi_{q}(\xi)$, i.e., $E=\exp \{i \beta \zeta+i \boldsymbol{q} \cdot \xi\} \varphi_{q}(\xi)$, reads

$$
\left[(\boldsymbol{q}-\boldsymbol{Q})^{2}-\beta\right] C_{\boldsymbol{q}-\boldsymbol{Q}}+\sum_{Q^{\prime}} \hat{V}_{\boldsymbol{Q}^{\prime}-\boldsymbol{Q}} C_{\boldsymbol{q}-\boldsymbol{Q}^{\prime}}=0
$$

where $Q$ denotes a vector of the reciprocal lattice and $C_{q-} Q$ and $\hat{V}_{Q^{\prime}-Q}$ are the Fourier components of the Bloch wave $\varphi_{q}(\xi)$ and the lattice $V(\xi)$, respectively. The probe beam with

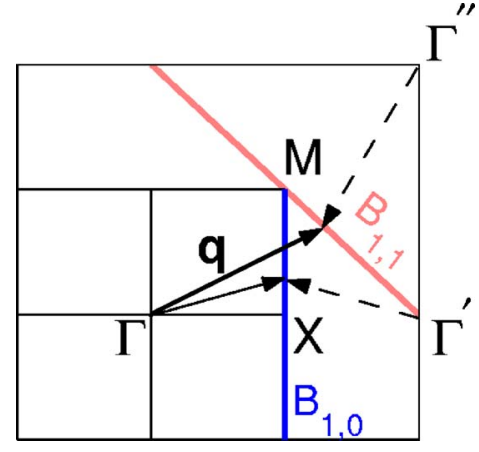

FIG. 2. (Color online) Schematic representation of the extended Brillouin zone and the two cases of the Bragg resonance for the two-dimensional optical lattice described in the text. Solid lines indicate the Bloch index and dashed lines are the resonant index given by $\boldsymbol{q}-\boldsymbol{Q}$ with $\boldsymbol{Q}$ corresponding to the considered Bragg plane.

the Bloch index $\boldsymbol{q}=(k, \ell)$ is effectively reflected by the lattice in the first order in $V_{0}$ when the end point of the Bloch index lies on the resonant Bragg plane defined by $\boldsymbol{Q}^{\prime}-\boldsymbol{Q}$, i.e., when $(\boldsymbol{q}-\boldsymbol{Q})^{2}=\left(\boldsymbol{q}-\boldsymbol{Q}^{\prime}\right)^{2}=\beta$ and $\hat{V}_{\boldsymbol{Q}^{\prime}-\boldsymbol{Q}} \neq 0$.

For the lattice (5), there are two distinct planes of the quasi-one dimensional Bragg resonance, denoted by $B_{1,0}$ and $B_{1,1}$ in Fig. 2. The corresponding vectors of the reciprocal lattice are $\boldsymbol{Q}=\left(2 q_{B}, 0\right)$ and $\boldsymbol{Q}=\left(2 q_{B}, 2 q_{B}\right)$, respectively (in our case, $\left.q_{B}=1 / 2\right)$. Below we consider the corresponding Bragg resonances.

\section{A. Generic $B_{1,0}$ resonance}

The reflection point on the Bragg plane $B_{1,0}$ is $\boldsymbol{q}=\left(q_{B}, \ell_{0}\right)$ with some $\ell_{0} \neq \pm q_{B}+O\left(V_{0}\right)$ (i.e., outside the neighborhood of the $M$ point of radius proportional to $V_{0}$ ). For a broad beam one can proceed in a way similar to the approach of Ref. [23], which studied accelerating electrons in the solid-state theory, and assume that the beam is described by a Bloch wave with the propagation-dependent Bloch index $\boldsymbol{q}=\boldsymbol{q}(\zeta)$. By keeping only the resonant terms we arrive at the following approximation for the Bloch wave describing a broad beam near the point of Bragg reflection (or, equivalently, resonance):

$$
\psi=\left[C_{1}(\zeta) e^{i k(\zeta) \xi_{1}}+C_{2}(\zeta) e^{i\left[k(\zeta)-2 q_{B}\right] \xi_{1}}\right] e^{i \ell(\zeta) \xi_{2}} .
$$

Substituting this expression into Eq. (1), with $x \Delta n$ $=V(\boldsymbol{\xi})+\boldsymbol{\alpha} \cdot \boldsymbol{\xi}$, and projecting on the resonant terms yields $d k / d \zeta=-\alpha_{1}, d \ell / d \zeta=-\alpha_{2}$, which are necessary to cancel the linear term in $\boldsymbol{\xi}$ (see, e.g., Ref. [17]), and then obtain a system of coupled equations for the incident $C_{1}$ and Bragg reflected $C_{2}$ amplitudes (see also Ref. [15]),

$$
\begin{gathered}
i \frac{d C_{1}}{d \zeta}=\frac{1}{2}\left(\ell^{2}+k^{2}\right) C_{1}+\frac{V_{0}}{2} C_{2}, \\
i \frac{d C_{2}}{d \zeta}=\frac{1}{2}\left(\ell^{2}+\left[k-2 q_{B}\right]^{2}\right) C_{2}+\frac{V_{0}}{2} C_{1} .
\end{gathered}
$$

It is convenient to set the propagation variable $\zeta$ equal to zero at the resonance point, so that 
$k=q_{B}-\alpha_{1} \zeta$. Redefining the amplitudes as $\left(C_{1}, C_{2}\right)$ $=\exp \left\{-i\left(\int^{\zeta} d \zeta \ell^{2}+q_{B}^{2} \zeta+\alpha_{1}^{2} \zeta^{3} / 3\right) / 2\right\}\left(c_{1}, c_{2}\right)$ we obtain the Landau-Zener-Majorana system [1-3]

$$
\begin{gathered}
i \frac{d c_{1}}{d \zeta}=-\frac{\alpha_{1} \zeta}{2} c_{1}+\frac{V_{0}}{2} c_{2}, \\
i \frac{d c_{2}}{d \zeta}=\frac{\alpha_{1} \zeta}{2} c_{2}+\frac{V_{0}}{2} c_{1} .
\end{gathered}
$$

System (9) is Hamiltonian; its adiabatic energy levels or, equivalently, the two Bloch bands of $\beta, \quad \beta_{1,2}$ $=\mp \sqrt{q^{2}+\left(V_{0} / 2\right)^{2}}$, experience an avoided crossing at $q=0$, where $q \equiv \alpha_{1} \zeta / 2$ has the meaning of the running band parameter.

The probability of tunneling, $P$, defined through the final amplitude $P=\left|C_{j}(\infty)\right|^{2}$ for the initial condition $\left|C_{j}(-\infty)\right|=1$, is given by the well-known Landau-Zener formula $P=\exp \left\{-\pi V_{0}^{2} /\left(2\left|\alpha_{1}\right|\right)\right\}$ for both the lower-to-upper and upper-to-lower band tunneling processes. Here $\alpha_{1}$ is the component of $\boldsymbol{\alpha}$ in the direction perpendicular to the Bragg plane $B_{1,0}$, i.e., to the $X M$ border of the irreducible Brillouin zone. In Fig. 1 this case of tunneling corresponds to the transitions between the two lowest bands along the $X M$-line. Thus, this case of interband tunneling is quasi-onedimensional even if it occurs in a two-dimensional lattice (e.g., see Refs. [15-17]).

\section{B. Generic $B_{1,1}$ resonance}

In this case, the reflection point on the Bragg plane $B_{1,1}$ is $\boldsymbol{q}=\left(k_{0}, \ell_{0}\right)$ with $k_{0}+\ell_{0}=2 q_{B}$ and $\ell_{0} \neq q_{B}+O\left(V_{0}\right)$. The resonant terms of the Bloch wave read

$$
\psi=C_{1}(\zeta) e^{i k(\zeta) \xi_{1}+i \ell(\zeta) \xi_{2}}+C_{2}(\zeta) e^{i\left[k(\zeta)-2 q_{B}\right] \xi_{1}+i\left[\ell(\zeta)-2 q_{B}\right] \xi_{2}}
$$

Setting $k=k_{0}-\alpha_{1} \zeta$ and $\ell=\ell_{0}-\alpha_{2} \zeta$, redefining the amplitudes as $\left(C_{1}, C_{2}\right)=\exp \left\{-i\left(\left[k_{0}^{2}+\ell_{0}^{2}+\left(\alpha_{2}-\alpha_{1}\right)\left(k_{0}-\ell_{0}\right)\right] \zeta\right.\right.$ $\left.\left.+\left[\alpha_{1}^{2}+\alpha_{2}^{2}\right] \zeta^{3} / 3\right) / 2\right\}\left(c_{1}, c_{2}\right)$, and following all the steps described above results in the following Landau-ZenerMajorana system:

$$
\begin{gathered}
i \frac{d c_{1}}{d \zeta}=-\frac{\Omega_{1} \zeta}{2} c_{1}+\frac{\varepsilon V_{0}}{4} c_{2}, \\
i \frac{d c_{2}}{d \zeta}=\frac{\Omega_{1} \zeta}{2} c_{2}+\frac{\varepsilon V_{0}}{4} c_{1},
\end{gathered}
$$

where $\Omega_{1}=\alpha_{1}+\alpha_{2}$.

The corresponding Bloch bands are $\beta_{1,2}$ $=\mp \sqrt{q^{2}+\left(\varepsilon V_{0} / 4\right)^{2}}$, with $q \equiv \Omega_{1} \zeta / 2$. The interband tunneling probability when crossing the $B_{1,1}$ plane is thus given by the formula $P=\exp \left\{-\pi \varepsilon^{2} V_{0}^{2} /\left(8\left|\alpha_{1}+\alpha_{2}\right|\right)\right\}$.

The Bragg plane $B_{1,1}$ is transformed to the $\Gamma M$ border of the irreducible Brillouin zone by the following transformation: $k=2 q_{B}-k^{\prime}, \ell=\ell^{\prime}$. Note that the transformation changes the coordinates as follows $\xi_{1}=-\xi_{1}^{\prime}, \xi_{2}=\xi_{2}^{\prime}$, thus $\alpha_{1}=-\alpha_{1}^{\prime}$ and $\alpha_{2}=\alpha_{2}^{\prime}$. Therefore, the probability of tunneling when cross- ing the $\Gamma M$ border of the irreducible Brillouin zone reads $P=\exp \left\{-\pi \varepsilon^{2} V_{0}^{2} /\left(8\left|\alpha_{2}-\alpha_{1}\right|\right)\right\}$.

The quantity $\left|\alpha_{2}-\alpha_{1}\right| / 2$ is the component of $\boldsymbol{\alpha}$ perpendicular to the $\Gamma M$ line (exactly $\left|\alpha_{2}-\alpha_{1}\right| / \sqrt{2}$ ) multiplied by the half length of the respective reciprocal lattice vector $\sqrt{2} q_{B}$. In this respect, this case of tunneling is again quasione-dimensional.

\section{Fourfold $B_{1,1}$ resonance}

In this more general case, the reflection point is the $M$ point. There exist exactly four Bloch indices which are in resonance; they correspond to the four high-symmetry points of the first Brillouin zone equivalent to the $M$ point. This case is specific to the two-dimensional tunneling, since it leads to a four-level system. The resonant part of the Bloch wave reads

$$
\begin{aligned}
\psi= & C_{1}(\zeta) e^{i k(\zeta) \xi_{1}+i \ell(\zeta) \xi_{2}}+C_{2}(\zeta) e^{i\left[k(\zeta)-2 q_{B}\right] \xi_{1}+i\left[\ell(\zeta)-2 q_{B}\right] \xi_{2}} \\
& +C_{3}(\zeta) e^{i\left[k(\zeta)-2 q_{B}\right] \xi_{1}+i \ell(\zeta) \xi_{2}}+C_{4}(\zeta) e^{i k(\zeta) \xi_{1}+i\left[\ell(\zeta)-2 q_{B}\right] \xi_{2}}
\end{aligned}
$$

Setting $k=q_{B}-\alpha_{1} \zeta$ and $\ell=q_{B}-\alpha_{2} \zeta$, defining $c_{j}$ $=e^{i\left(q_{B}^{2} \zeta+\left[\alpha_{1}^{2}+\alpha_{2}^{2}\right] \zeta^{3} / 6\right)} C_{j}$, and following all the above steps we derive the following system:

$$
\begin{gathered}
i \frac{d c_{1}}{d \zeta}=-\frac{\Omega_{1} \zeta}{2} c_{1}+\frac{\varepsilon V_{0}}{4} c_{2}+\frac{V_{0}}{2}\left(c_{3}+c_{4}\right), \\
i \frac{d c_{2}}{d \zeta}=\frac{\Omega_{1} \zeta}{2} c_{2}+\frac{\varepsilon V_{0}}{4} c_{1}+\frac{V_{0}}{2}\left(c_{3}+c_{4}\right), \\
i \frac{d c_{3}}{d \zeta}=-\frac{\Omega_{2} \zeta}{2} c_{3}+\frac{\varepsilon V_{0}}{4} c_{4}+\frac{V_{0}}{2}\left(c_{1}+c_{2}\right) \\
i \frac{d c_{4}}{d \zeta}=\frac{\Omega_{2} \zeta}{2} c_{4}+\frac{\varepsilon V_{0}}{4} c_{3}+\frac{V_{0}}{2}\left(c_{1}+c_{2}\right)
\end{gathered}
$$

with $\Omega_{1}=\alpha_{1}+\alpha_{2}$ and $\Omega_{2}=\alpha_{2}-\alpha_{1}$. The invariance of the system with respect to rotations by $\pi / 2$, which is the invariance of the lattice itself, is evident from the corresponding transformation: $\alpha_{1} \rightarrow \alpha_{2}$ and $\alpha_{2} \rightarrow-\alpha_{1}$.

The system (13)-(16) can be viewed as a coupling (by the terms with $V_{0} / 2$ ) of two cases of the Bragg reflection: one is for crossing of the $B_{1,1}$ plane [Eqs. (13) and (14)] and the other one is for crossing of the $B_{-1,1}$ plane [Eqs. (15) and (16); the reciprocal lattice vector is $\left.\boldsymbol{Q}=\left(-2 q_{B}, 2 q_{B}\right)\right]$.

A schematic correspondence between the amplitudes $\left|c_{k}\right|^{2}$ of the Bloch wave and the tunneled and reflected beams is depicted in Fig. 3.

The generalized (i.e., multilevel) Landau-Zener-Majorana system defined by Eqs. (13)-(16) leads to a fourth-order polynomial which does not allow a simple analytical solution for the Bloch bands. However, it is easy to establish that the four Bloch bands assume the following values at the crossing point $(\zeta=0)$ : 


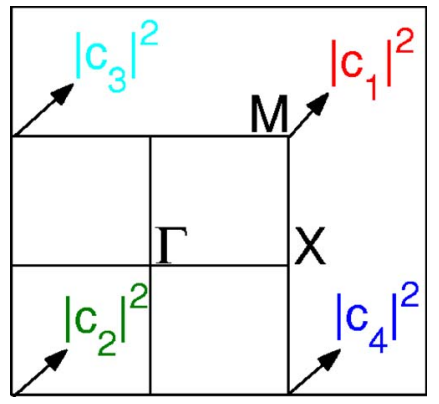

FIG. 3. (Color online) Schematic representation of tunneling at the $M$ point of the Brillouin zone. The four beams follow the direction of the linear ramp (indicated by arrows; here along the ГM line).

$$
\beta(M)=\frac{1}{4}+V_{0}\left[\frac{\varepsilon}{4}-1 ;-\frac{\varepsilon}{4} ;-\frac{\varepsilon}{4} ; 1+\frac{\varepsilon}{4}\right],
$$

where we have used that $\left|\boldsymbol{q}_{M}\right|=1 / \sqrt{2}$. Thus, recalling that in our case $\varepsilon=1$, we see that the shallow lattice captures at least the qualitative behavior of the Bloch bands at the $M$ point of the experimental two-dimensional optical lattice of Ref. [11], where the two inner Bloch bands are also crossing at the $M$ point (see also Fig. 1).

Though there exists no general formula describing the tunneling probabilities between various levels in a multilevel Landau-Zener-Majorana system (see, e.g., Refs. [24-26] for further discussion), in the generic case, i.e., $\alpha_{1,2} \neq 0$, the probability of the transition between the lowest and the highest adiabatic levels is known [24,26]. This corresponds to the tunneling across the complete band gap discussed in Ref. [11]. Assuming that $\left|\alpha_{1}+\alpha_{2}\right|>\left|\alpha_{2}-\alpha_{1}\right|$, we obtain the probability of tunneling between the first and the fourth bands as follows:

$$
P=\exp \left\{-\frac{\pi V_{0}^{2}}{2}\left[\frac{\varepsilon^{2}}{4\left|\alpha_{2}+\alpha_{1}\right|}+\frac{1}{\left|\alpha_{1}\right|}+\frac{1}{\left|\alpha_{2}\right|}\right]\right\}
$$

In the opposite case, i.e., when $\left|\alpha_{1}+\alpha_{2}\right|<\left|\alpha_{2}-\alpha_{1}\right|$, the Bloch index passes through an equivalent $M$ point and the probabil-

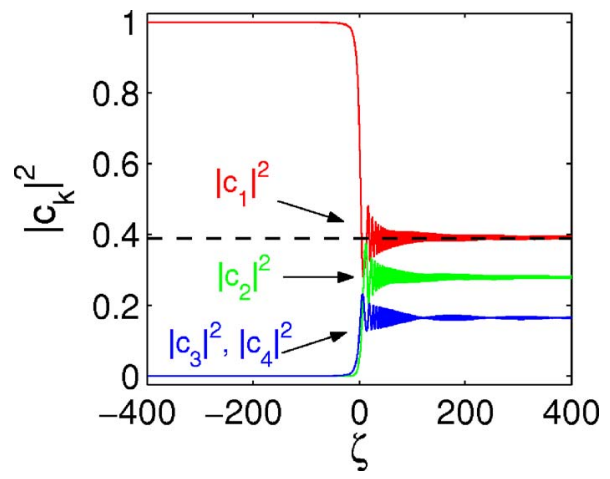

FIG. 4. (Color online) Tunneling at the $M$ point of the Brillouin zone. Here the parameters are $\alpha=0.05[\cos (\pi / 4), \sin (\pi / 4)]$, i.e., the ramp is in the $\Gamma M$ direction, $V_{0}=0.1$, and $\varepsilon=1$. The dashed line is the analytical prediction given by the formula (18).

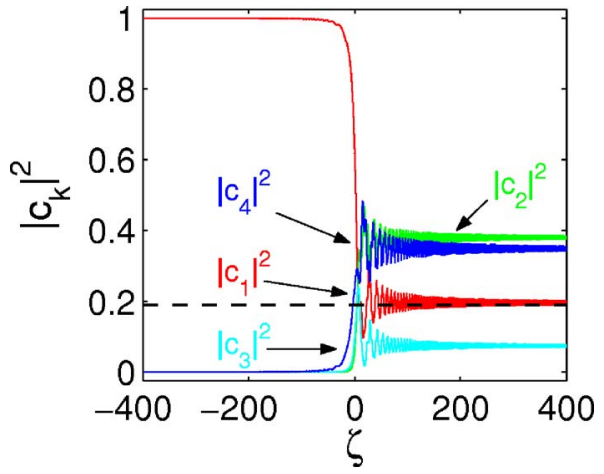

FIG. 5. (Color online) Tunneling at the $M$ point. Here the parameters are $V_{0}=0.1, \varepsilon=1$, and $\boldsymbol{\alpha}=0.05[\cos (0.25)$, $\sin (0.25)]$, i.e., the ramp is directed close to the $\Gamma X$ line. The dashed line is the analytical prediction.

ity of tunneling is given by the same formula with the change $\left|\alpha_{1}+\alpha_{2}\right| \rightarrow\left|\alpha_{2}-\alpha_{1}\right|$.

Two particular cases of tunneling at the $M$ point are shown in Figs. 4 and 5. We have simulated numerically the system (13)-(16) using the fourth-order Runge-Kutta method. Since the system is written for the so-called diabatic basis, a large $\zeta$ interval was used for reliable results. Asymptotically, i.e., for $\zeta \rightarrow \pm \infty$, the diabatic basis coincides with the adiabatic one (in our case, the latter defines the respective Bloch bands). The dashed line gives the analytical prediction of Eq. (18). The oscillations of the amplitudes $\left|c_{k}\right|^{2}$ in Figs. 4 and 5 after the tunneling process reflect a slight difference between the respective amplitudes in the diabatic and adiabatic bases (in the adiabatic basis there are no oscillations).

In Fig. 4 the ramp direction is along the $\Gamma M$ line and the output powers of the side beams (i.e., $W \propto\left|C_{3,4}\right|^{2}$ ) are the same. In Fig. 5 the ramp direction is close to the $\Gamma X$ border, thus creating a substantial asymmetry in the output beams 3 and 4 , similar to the tunneling observed in the experiment [11].

We conclude this a section with a discussion of validity of the Landau-Zener-Majorana models considered above. In particular, we consider the fourfold $B_{1,1}$ resonance. The sys-

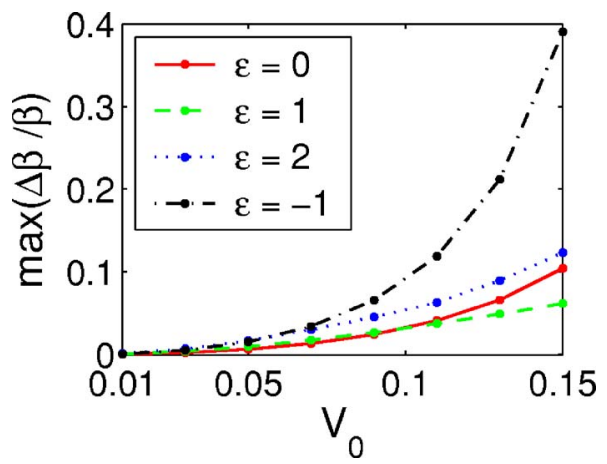

FIG. 6. (Color online) The error of the Landau-Zener-Majorana model for the fourfold $B_{1,1}$ resonance. The curves give the absolute value of the maximal relative difference between the first four Bloch bands of the full two-dimensional equation and the corresponding energy levels of the four-level Landau-Zener-Majorana model. 
tem (13)-(16) approximates the four resonant Bloch bands of the lattice at the $M$ point (see Fig. 1). The approximate analytical values for the four energies at the $M$ point, given by Eq. (17), can be employed to derive a quantitative estimate on the validity of the Landau-Zener-Majorana model (13)-(16). The result is presented in Fig. 6. We have used four values of the parameter $\varepsilon: \varepsilon=0$ (solid line; the egg crate, i.e., separable lattice), 1 (dashed line; the quantum antidot in the terminology of Ref. [13]), 2 (dotted line), and -1 (dash-dotted line; the quantum dot).

From Fig. 6 one can see that for the lattice potentials with $V_{0} \leq 0.05$ the relative error of the finite-dimensional approximation of Zener tunneling by the Landau-Zener-Majorana models is below 5\%. For the experimental lattice of Ref. [11], i.e., the quantum antidot $(\varepsilon=1)$, the relative error is less then $5 \%$ for $V_{0} \leq 0.15$. On the other hand, for any particular lattice potential, for the values of the lattice strength $V_{0}$ above some threshold the finite-dimensional approximation will break down, as it does for the quantum dot lattice already for $V_{0}=0.15$.

\section{CONCLUDING REMARKS}

We have derived simple models that allow one to describe one-dimensional and more general two-dimensional Zener tunneling in two-dimensional periodic photonic structures and calculate the corresponding tunneling probabilities.

We have found that a square two-dimensional photonic lattice allows one to observe the quasi-one-dimensional tunneling, when the Bloch index crosses either the $\Gamma M$ border or the $X M$ border of the irreducible Brillouin zone away from the $M$ point, and the specific two-dimensional tunneling, when the Bloch index passes through the border of the Brillouin zone at the $M$ point (more precisely, in its neighborhood with radius of the order of $V_{0}$ ). We notice that in the first order in $V_{0}$ the Bloch oscillations are not affected by tunneling when the Bloch index crosses the $\Gamma X$ border of the irreducible Brillouin zone of the two-dimensional lattice given by Eq. (5) of Sec. II. We have considered only one possible case of the genuine two-dimensional tunneling at the $M$-point, but it can be also observed at the $\Gamma$-point. This case, however, is more difficult to realize experimentally, since the beam should be initially prepared in the higher-band.

In our analytical calculations we have employed a shallow-lattice approximation which can be related to the experimental setup used in Ref. [11] only qualitatively, and we do observe at least qualitative correspondence with the experiment. There exist two general ways to realize a shallow lattice in the experimental setup. The first one is to reduce the power of the laser beams creating the optical lattice potential $I_{g}(\xi)$; this requires the condition $A^{2} \ll 1 / \varkappa$, which we have adopted in this paper. The second one is to change the value of the parameter $x=\gamma n_{0}(d / \lambda)^{2}$ so that $x \ll 1$. In the latter case, the lattice potential will be different from that used in our analytic calculations [see Eq. (5)], namely, it will have all higher harmonics in the Fourier expansion. Qualitatively this will result in the following changes. First of all, there will be a substantial tunneling when crossing the $\Gamma X$ border. Second, the higher harmonics will also affect the probabilities of all other tunneling cases. However, the latter effect is of a higher order in the potential amplitude, since the higher harmonics are not resonant. Therefore, the theory developed here can be applied to this case as well. The tunneling when crossing the $\Gamma X$ border is quasi-onedimensional, and it can be treated similarly to the quasi-onedimensional tunneling cases discussed in this paper.

\section{ACKNOWLEDGMENTS}

This work was supported by a CNPq-FAPEAL grant in Brazil and the Australian Research Council in Australia. Y.K. thanks Anton Desyatnikov for useful discussions of the applicability of the shallow-lattice approximation to the experimental conditions.
[1] C. Zener, Proc. R. Soc. London, Ser. A 137, 696 (1932).

[2] L. D. Landau, Phys. Z. Sowjetunion 2, 46 (1932).

[3] E. Majorana, Nuovo Cimento 9, 43 (1932).

[4] L. Esaki, Rev. Mod. Phys. 46, 237 (1974).

[5] B. Bourlon, D. C. Glattli, B. Placais, J. M. Berroir, C. Miko, L. Forro, and A. Bachtold, Phys. Rev. Lett. 92, 026804 (2004).

[6] A. Sibille, J. F. Palmier, and F. Laruelle, Phys. Rev. Lett. 80, 4506 (1998).

[7] G. Ithier, E. Collin, P. Joyez, D. Vion, D. Esteve, J. Ankerhold, and H. Grabert, Phys. Rev. Lett. 94, 057004 (2005).

[8] M. Jona-Lasinio, O. Morsch, M. Cristiani, N. Malossi, J. H. Müller, E. Courtade, M. Anderlini, and E. Arimondo, Phys. Rev. Lett. 91, 230406 (2003).

[9] M. Jona-Lasinio, O. Morsch, M. Cristiani, E. Arimondo, and C. Menotti, Laser Phys. 15, 1180 (2005).

[10] H. Trompeter, T. Pertsch, F. Lederer, D. Michaelis, U. Streppel, A. Bräuer, and U. Peschel, Phys. Rev. Lett. 96, 023901
(2006).

[11] H. Trompeter, W. Krolikowski, D. N. Neshev, A. S. Desyatnikov, A. A. Sukhorukov, Yu. S. Kivshar, T. Pertsch, U. Peschel, and F. Lederer, Phys. Rev. Lett. 96, 053903 (2006).

[12] A. R. Kolovsky and H. J. Korsch, Phys. Rev. A 67, 063601 (2003).

[13] D. Witthaut, F. Keck, H. J. Korsch, and S. Mossmann, New J. Phys. 6, 41 (2004).

[14] V. A. Brazhnyi, V. V. Konotop, and V. Kuzmiak, Phys. Rev. Lett. 96, 150402 (2006).

[15] B. Wu and Q. Niu, Phys. Rev. A 61, 023402 (2000).

[16] O. Zobay and B. M. Garraway, Phys. Rev. A 61, 033603 (2000).

[17] V. S. Shchesnovich and S. B. Cavalcanti, J. Phys. B 39, 1997 (2006).

[18] It is well known that for a strong linear potential the Bloch- 
band structure is replaced by the set of Wannier-Stark states [19]; see also the discussion in Ref. [20].

[19] G. H. Wannier, Phys. Rev. 117, 432 (1960).

[20] M. Glück, F. Keck, A. R. Kolovsky, and H. J. Korsch, Phys. Rev. Lett. 86, 3116 (2001).

[21] J. M. Ziman, The Principles of the Theory of Solids (Cambridge University Press, Cambridge, U.K., 1972).
[22] N. W. Ashcroft and N. D. Mermin, Solid State Physics (Holt, Rinehart and Winston, New York, 1976).

[23] W. V. Houston, Phys. Rev. 57, 184 (1940).

[24] S. Brundobler and V. Elser, J. Phys. A 26, 1211 (1993).

[25] Yu. N. Demkov and V. N. Ostrovsky, Phys. Rev. A 61, 032705 (2000).

[26] A. V. Shytov, Phys. Rev. A 70, 052708 (2004). 